\title{
Racial Disparities in Treatment Utilization for Multiple Myeloma
}

\author{
Mark A. Fiala, BS, CCRP ${ }^{1,2}$ and Tanya M. Wildes, MD, MSCI ${ }^{1}$ \\ ${ }^{1}$ Division of Oncology, Department of Medicine, Washington University School of Medicine, St. \\ Louis MO \\ ${ }^{2}$ George Warren Brown School of Social Work, Washington University, St. Louis MO
}

\begin{abstract}
Background-Recent treatment advances have greatly improved the prognosis of patients with multiple myeloma. However, some of these newer, more effective treatments are intensive and expensive and their utilization is still low, particularly among black patients.
\end{abstract}

Methods-In this study, we reviewed the utilization patterns of stem cell transplantation and bortezomib using the Surveillance, Epidemiology, and End Results (SEER)-Medicare linked database.

Results-After controlling for overall health and potential access barriers, black patients were $37 \%$ ( $\mathrm{p}<0.0001$ ) less likely to utilize stem cell transplantation, and $21 \%$ ( $\mathrm{p}<0.0001$ ) less likely to utilize bortezomib. Moreover, we found that the underutilization of these agents was the associated with a $12 \%(p=0.0007)$ increase in hazard ratio for death among black patients.

Conclusion-Eliminating health disparities, a current focus of U.S. public policy, is highly complex, as illustrated by the results of this study. In patients with multiple myeloma, treatment disparities are not completely explained by potential access barriers. Additional factors, such as structural barriers in the health care system and individual decision-making among black and white patients, must be explored to fully explain the disparity.

\section{Keywords}

Cancer disparities; race; access barriers; stem cell transplantation; SEER-Medicare; multiple myeloma

\section{Introduction}

Treatment advances over the past 2 decades have greatly improved the survival of patients with multiple myeloma (MM). ${ }^{1}$ However, some of these newer, more effective treatments are often much more intensive and expensive than other treatment options and their utilization on the population level is low. For example, only approximately $13 \%$ of MM patients undergo autologous stem cell transplantation (ASCT). ${ }^{2}$

Corresponding author: Mark A. Fiala, BS CCRP, 660 S. Euclid Avenue, Campus Box 8056, St. Louis MO 63110, USA, Phone: 314-454-8302, Fax: 314-273.0580, mfiala@dom.wustl.edu.

Disclosures: The authors have no conflicts of interest to disclose.

Author Contributions: Both authors contributed to all aspects of the research, analysis, and manuscript preparation. 
Utilization of ASCT also varies based on patient demographics. Older patients and racial/ ethnic minorities are less likely to undergo ASCT., ${ }^{3,4}$ Older patients are more likely to have comorbid conditions or other contraindications to the procedure and, until recently, the procedure was largely reserved for patients under 78 years of age in part due to Medicare coverage guidelines. ${ }^{5}$ These factors could account for the age-related disparity. The cause of the racial disparity is less clear. Researchers initially attributed it to access barriers such as low socioeconomic status (SES) and/or inadequate health insurance; ${ }^{4}$ both have been linked to poorer survival in MM. ${ }^{6}$ However, our group recently reported that black patients treated at our institution were 54\% less likely to undergo ASCT after controlling for potential access barriers. $^{7}$

In this study, we aimed to confirm our previous finding on racial disparities in ASCT utilization among patients with $\mathrm{MM}$ in a nationally representative sample. We also reviewed the utilization patterns of bortezomib among white and black patients, a topic not reported to date. We chose bortezomib as a comparator as it has largely become the standard of care in recent years and, unlike ASCT, bortezomib is not exclusive to specialized centers; it can be administered at any facility that administers chemotherapy. We then analyzed how the utilization patterns of these two treatments impact outcome disparities in MM.

\section{Methods}

Data Source

The source of data for this study was the National Cancer Institute's (NCI) Surveillance, Epidemiology, and End Results (SEER)-Medicare linked database. SEER collects demographics, tumor characteristics, and survival data from 18 population-based cancer registries throughout the United States, covering approximately $26 \%$ of the U.S. Population including $23 \%$ of African-Americans. ${ }^{8}$ In the SEER-Medicare linked database, the SEER registry data is linked to Medicare enrollment and claims data. Of all people 65 years of age or older in the SEER registry, 93\% have been matched to their corresponding Medicare data. ${ }^{8}$ The SEER-Medicare database has been described in detail elsewhere. ${ }^{9}$ At the time this study was conducted, the SEER-Medicare linkage included all Medicare-eligible persons appearing in the SEER data through 2011 and their Medicare claims through 2013.

The following Medicare claims files were used for this analysis: Medicare Provider Analysis and Review (MEDPAR), which includes all hospital short-stay, long-stay, and skilled nursing facility bills [Medicare Part A]; National Claims History (NCH), which includes all physician/supplier bills [Part B]; Outpatient, which includes all bills from institutional outpatient providers [Part B]; Home Health Agency (HHA), which includes all claims for home health services [Part B]; and Durable Medical Equipment (DME), which includes all claims for durable medical equipment such as oxygen tanks [Part B].

\section{Inclusion and Exclusion Criteria}

In total, 46,328 patients diagnosed with MM between 2000 and 2011 were available in the SEER-Medicare database. Identification of MM was made using the World Health Organization (WHO) International Classification of Diseases for Oncology, 3rd Edition 
(ICD-O-3) histology codes 9731-9732, and 9734, which were all recoded to 34000 in the SEER database. ${ }^{10}$

We excluded cases with duplicate or incomplete records including death certificate or autopsy cases, cases not enrolled in Medicare Part A and Part B at diagnosis, and managed care (HMO) enrollees. We also excluded all cases that did not have $\geq 1$ claim within one year prior to diagnosis as this is another indicator of a possible incomplete record. We also excluded cases where reported race was not white or black or where MM was diagnosed prior to age 65. Medicare eligibility prior to age 65 is restricted to those with severe illness or disability and thus these patients may not be generalizable. This left 20,916 patients for the analyses. Supplementary Figure 1 details the screening flow chart.

The SCT utilization analysis was limited to patients who were $<78$ years old at MM diagnosis as coverage for older patients was previously restricted as described above. The BTZ utilization analysis was limited to patients diagnosed after 2003 to coincide with its FDA approval. The patients who were included in both the SCT and BTZ utilization analyses were included in the survival analysis.

\section{Variables}

Treatment utilization was determined using the Medicare claims data $(\mathrm{NCH}$ and OUTPATIENT) with the International Classification of Diseases (9th revision), Clinical Modification (ICD-9-CM) diagnosis and procedure codes which are used to identify claims for treatments or procedures. ${ }^{11}$

The presence or absence of the corresponding procedure codes for ASCT (HCPCS 38241 or ICD-9 codes 41.00, 41.01, 41.04, 41.07, or 41.09) and allogeneic stem cell transplantation (HCPCS 38240 or 38242 , or ICD-9 codes 41.02, 41.03, 41.05, 41.06 or 41.08) were used to determine SCT utilization. Although allogeneic transplantation is uncommon among MM patients who have not previously failed ASCT, it is sometimes administered to younger patients, those with identical twins, or those who fail mobilization. ${ }^{12}$ Therefore, we included the codes for the procedure to prevent this from confounding the analyses. Bortezomib utilization was determined by the presence or absence of the HCPCS code J9041.

Patients were coded as black or white and male or female based on SEER data. Age at MM diagnosis and year of diagnosis were treated as continuous variables. We used socioeconomic status (SES), Medicaid enrollment, and geography to control for potential access barriers. Direct SES data at the patient level is not available through SEER-Medicare. Instead, the database contains information from the 2000 Census reported at the tract level in which the patient lived at diagnosis; the median household income (MHI), a continuous variable, was used to approximate SES. Patients enrolled in Medicaid at any time during the year of diagnosis were considered as enrolled. Geography was determined by the 2003 Rural/Urban Continuum Code from the Department of Agriculture's Economic Research Service for the county of home residence at diagnosis.

Performance status (PS) is not directly available in the database. In its absence, Medicare claims were used to identify several claims-based indicators of PS, including the use of 
oxygen and related respiratory therapy supplies, wheelchairs and supplies, home health agency use, and skilled nursing facility use within 12 months prior to MM diagnosis. PS was then coded as normal ( 0 indicators) or poor ( 1 or more indicators) as previously described. ${ }^{13}$

Comorbidities were not directly available in the database as well. Diagnosis and procedure codes from the Medicare claims data (MEDPAPR, $\mathrm{NCH}$, and OUTPATIENT) for the 12 months prior to MM diagnosis were used to calculate a Charlson Comorbidity Index (CCI) score for each patient using established algorithms. ${ }^{14,15}$

Overall survival was defined as the number of months from MM diagnosis to death from any cause. Patients still living were censored at the time of the data cutoff. Full details on variable coding can be found in Supplementary Table 1.

\section{Statistical Analysis}

Multivariate logistic regression models of ASCT and BTZ utilization were created in a stepwise fashion to determine how each set of variables influenced utilization. Initially only race, sex, and year of diagnosis were included in the model [Model 1]. Then overall health measures (age at diagnosis, PS, and CCI) were added [Model 2]. Lastly, potential access barriers were added (MHI, Medicaid, and geography) [Model 3].

Cox regression models of survival were then created in a step-wise fashion to determine how each set of variables impacted overall survival. Initially only race, sex, and year of diagnosis were included in the model [Model 1]. Then ASCT and BTZ utilization were added [Model 2]. Lastly overall health measures and potential access barriers were added [Model 3].

\section{Ethical Considerations}

This study was conducted as part of a research protocol approved by the Washington University Institutional Review Board (IRB). This specific analysis was exempt from IRB review as the data is not individually identifiable and, therefore, is not covered by 45 Code of Federal Regulations part 46 "Protection of Human Subjects" per the guidelines set forth by the Department of Health and Human Services. ${ }^{16}$

\section{Results}

Demographics are summarized in Table 1. A total of 20,916 patients met the eligibility criteria for analysis. Eighty-four percent $(\mathrm{n}=17,574)$ were white and $16 \%(\mathrm{n}=3,342)$ were black. Black patients were younger at diagnosis (75.9 years $[S D=7.0]$ compared to 77.3 years [ $S D=7.0] ; t=10.94, p<0.0001)$, but were more likely to have indicators of poor PS ( $27 \%$ compared to $\left.19 \% ; x^{2}=92.29, p<0.0001\right)$, and a CCI $\geq 1$ (59\% compared to $47 \% ; x^{2}$ $=223.98, p<0.001)$. Black patients also had lower MHI on average $(\$ 35,500[\mathrm{SD}=\$ 17,300]$ compared to $\$ 52,400$ [SD $=\$ 24,200] ; t=47.55, p<0.0001$ ) and were more likely to be Medicaid beneficiaries (34\% compared to $11 \% ; x^{2}=1120.89, p<0.0001$ ).

\section{SCT Utilization}

Fifty-four percent of patients $(n=11,269)$ were eligible for the SCT utilization analysis. Overall SCT utilization was low; only $7 \%$ of patients underwent the procedure. Utilization 
was higher among whites than blacks ( $8 \%$ compared to $\left.4 \% ; x^{2}=34.37, p<0.0001\right)$. In the initial regression model, blacks were $49 \%$ less likely to utilize SCT than whites ( $p<$ 0.0001 ). After controlling overall health, there was no change; blacks were $49 \%$ less likely to utilize SCT $(p<0.0001)$. After additionally controlling for potential access barriers (MHI, Medicaid, and urban/rural status), they were $37 \%$ less likely ( $p<0.0001)$. The model was significantly associated with SCT utilization $\left(x^{2}=508.02_{(10)}, p<0.0001\right)$. Results from the regression models of SCT utilization are summarized in Table 2.

\section{BTZ Utilization}

Seventy-seven percent of the whole cohort $(n=16,037)$ were eligible for the BTZ utilization analysis. Overall $36 \%$ of patients utilized BTZ. Utilization was higher among whites than blacks ( $36 \%$ compared to $30 \% ; x^{2}=39.10, \mathrm{p}<0.0001$ ). In the initial regression model, blacks were $24 \%$ less likely to utilize BTZ than whites ( $p<0.0001$ ). After controlling overall health, the disparity increased; blacks were $30 \%$ less likely $(p<0.0001)$. After additionally controlling for potential access barriers, they were $21 \%$ less likely $(p<0.0001)$. The model was significantly associated with BTZ utilization $\left(x^{2}=1733.10_{(10)}, p<0.0001\right)$. Results from the regression models of BTZ utilization are summarized in Table 3.

\section{Survival}

The survival analysis included 8,625 patients who were eligible for both the SCT and BTZ utilization analyses. At the time of data cutoff, $69 \%(n=6,013)$ of the patients had expired. Overall, the hazard ratio of black patients was $12 \%$ higher than whites $(p=0.0007)$. After controlling for SCT and BTZ utilization, there was no significant $(p=0.1124)$ difference in hazard ratio between the two groups. When overall health and potential access barriers were added the hazard ratio of black patients was $9 \%$ lower than whites $(\mathrm{p}=0.0068)$. The model was significantly associated with survival $\left(x^{2}=1026.66_{(12)}, \mathrm{p}<0.0001\right)$. Results from the Cox regression survival models of are summarized in Table 4.

\section{Discussion}

In this study, we found that disparities in treatment utilization for MM persist despite controlling for potential access barriers and overall health status. In our previous study of disparities in SCT utilization, blacks were 51\% less likely to utilize SCT compared to 37\% in the current study. ${ }^{7}$ The differences in results could be due to variations in methodology, such as patient selection (the previous study included patients of all ages, the median age was 59 years at diagnosis), or could represent differences in patients treated at a NCIdesignated Comprehensive Cancer Center versus the population in general.

The effects of overall health and potential access barriers on the disparities in SCT and BTZ utilization were very similar. After controlling for overall health, there was no change in underutilization of SCT among blacks, but there was an increase in the underutilization of BTZ. Although, black patients in this study were more likely to have indicators of poor PS and a higher CCI, they were also younger on average; therefore, differences in overall health did not explain the disparity in treatment utilization. Potential access barriers explained only a small portion of the underutilization of SCT and BTZ. 
The similar results between SCT and BTZ indicate that: 1) the disparity is not treatment specific; and 2) the disparity is less likely due to referral bias. As noted above, while SCT is an intensive procedure that often requires inpatient hospitalization for weeks at a specialized facility that offers the procedure, bortezomib can be administered on an outpatient basis in virtually any facility that administers chemotherapy.

While structural barriers in the health care system (referral bias, cultural barriers, a lack of coordination of care, or conscious or unconscious bias among physicians) likely contribute to treatment disparities in MM, we hypothesize that differences in medical decision making between black and white patients may also be involved. Factors such as personal preferences or mistrust of the medical system (conscious or unconscious) may contribute to higher rates of black patients receiving inferior treatment. ${ }^{17}$ Individual decision-making is often overlooked in studies of cancer treatment disparities. However, we believe that this is a pivotal aspect as each patient at least in part determines his/her selected treatment course.

A strength of this study is its generalizability, as it is based on a nationally representative sample. However, there are several inherent limitations of studies using observational data. First, racial and ethnic minorities are often underrepresented in available datasets. The SEER database covers approximately $23 \%$ of African-Americans, but black patients only represented $16 \%$ in our study. In the SEER database, the average age at MM diagnosis is 65 for blacks compared to 70 for whites. ${ }^{18}$ Therefore, black patients in SEER are less likely to be Medicare eligible, and thus blacks are underrepresented in the SEER-Medicare database. Our previous study of SCT utilization included patients of all ages and insurance providers and results were similar to the current study, therefore, we do not think the underrepresentation impacted the findings.

Also, there is a small minority of MM patients with asymptomatic or "smoldering" disease that do not require treatment and there is currently no validated claims-based method to identify them. However, the incidence of asymptomatic MM is similar between white and black patients and, therefore, should not confound comparisons of racial differences. ${ }^{19}$ Another limitation of this analysis is the lack of direct measures of SES and PS, and the use of surrogates for these measures. In addition, the database lacks any proxy variable for stem cell mobilization success. Therefore, it is possible that inferior stem cell mobilization among blacks could, in part, explain this disparity in ASCT utilization. This is not likely though, as black race has been associated with improved mobilization compared to whites. ${ }^{20}$

Despite the limitations, this study highlights the complexity of health disparities. In patients with MM, treatment disparities are not completely explained by variations in overall health and potential access barriers. We hypothesize that structural barriers in the health care system and differences in individual decision-making among black and white patients likely contribute to the disparity.

\section{Supplementary Material}

Refer to Web version on PubMed Central for supplementary material. 


\section{Acknowledgments}

Thank you to Dr. Shanta Pandey, Yu-Chih Chen, and the members of the George Warren Brown School of Social Work Research Specialization 2015-2016 cohort for their collaboration and input. Thank you to James Dukeman for assistance with SAS coding and to Dr. Robert Griffiths for providing the ICD-9 codes used as performance status indicators.

Dr. Wildes' research is supported by Grant Number 1K12CA167540 through the National Cancer Institute (NCI) at the National Institutes of Health (NIH) and Grant Number UL1 TR000448 through the Clinical and Translational Science Award (CTSA) program of the National Center for Advancing Translational Sciences (NCATS) at the National Institutes of Health. Its contents are solely the responsibility of the authors and do not necessarily represent the official view of NCI, NCATS or NIH.

This study used the linked SEER-Medicare database. The interpretation and reporting of these data are the sole responsibility of the authors. The authors acknowledge the efforts of the Applied Research Program, National Cancer Institute; the Office of Research, Development and Information, Centers for Medicare \& Medicaid Services; Information Management Services Inc; and the SEER program tumor registries in the creation of the SEERMedicare database.

\section{References}

1. Moreau P, Attal M, Facon T. Frontline therapy of multiple myeloma. Blood. 2015; 125(20):30763084. [PubMed: 25838345]

2. Costa LJ, Zhang MJ, Zhong X, Dispenzieri A, Lonial S, Krishnan A, Hari PN. Trends in utilization and outcomes of autologous transplantation as early therapy for multiple myeloma. Biol Blood Marrow Transplant. 2013; 19(11):1625-1624. [PubMed: 24035782]

3. Winn AN, Shah GL, Cohen JT, Lin PJ, Parsons SK. The real world effectiveness of hematopoietic transplant among elderly individuals with multiple myeloma. J Natl Cancer Inst. 2015; 107(8)doi: 10.1093/jnci/djv139

4. Costa LJ, Huang JX, Hari PN. Disparities in utilization of autologous hematopoietic cell transplantation for treatment of multiple myeloma. Biol Blood Marrow Transplant. 2015; 21(4): 701-706. [PubMed: 25555447]

5. Department of Health and Human Services Health Care Financing Administration. Medicare Intermediary Manual: Part 3-Claims Process: Transmittal 1805. 2000. Available from https:// www.cms.gov/Regulations-and-Guidance/Guidance/Transmittals/downloads/R1805A3.pdf. Retrieved November 11, 2016.

6. Fiala MA, Finney JD, Liu J, Stockerl-Goldstein KE, Tomasson MH, Vij R, Wildes TM. Socioeconomic Status is Independently Associated with Overall Survival in Patients with Multiple Myeloma. Leuk Lymphoma. 2015a; 56(9):2643-2649. [PubMed: 25651424]

7. Fiala MA, Finney JD, Stockerl-Goldstein KE, Tomasson MH, DiPersio JF, Vij R, Wildes TM. Re: Disparities in Utilization of Autologous Hematopoietic Cell Transplantation for Treatment of Multiple Myeloma. Biol Blood Marrow Transplant. 2015b; 21(7):1153-1154. [PubMed: 25771403]

8. National Cancer Institute. About the SEER Program. 2015b. Available from: http://seer.cancer.gov/ about/. Accessed November 8, 2015

9. Warren JL, Klabunde CN, Schrag D, Bach PB, Riley GF. Overview of the SEER-Medicare Data: Content, Research Applications, and Generalizability to the United States Elderly Population. Medical Care. 2002; 40(8):IV3-IV18.

10. North American Association of Central Cancer Registries. Guidelines for ICD-O-3 Implementation. 2015. Available from: www.facs.org/cancer/coc/naaccr.pdf. Accessed November 8, 2015.

11. Centers for Disease Control and Prevention. International Classification of Diseases, Ninth Revision, Clinical Modification (ICD-9CM). 2013. Available from: http://www.cdc.gov/nchs/icd/ icd9cm.htm. Accessed November 8, 2015

12. Bensinger W, Rotta M, Storer B, Chauncey T, Holmberg L, Becker P, Maloney D. Allo-SCT for multiple myeloma: a review of outcomes at a single transplant center. Bone Marrow Transplant. 2012; 47(10):1312-1317. [PubMed: 22327126] 
13. Griffiths R, Mikhael J, Gleeson M, Danese M, Dreyling M. Addition of rituximab to chemotherapy alone as first-line therapy improves overall survival in elderly patients with mantle cell lymphoma. Blood. 2011; 118(18):4808-4816. [PubMed: 21873544]

14. Charlson ME, Pompei P, Ales KL, MacKenzie CR. A new method of classifying prognostic comorbidity in longitudinal studies: Development and validation. Journal of Chronic Diseases. 1987; 40(5):373-383. [PubMed: 3558716]

15. Klabunde CN, Potosky AL, Legler JM, Warren JL. Development of a comorbidity index using physician claims data. Journal of clinical epidemiology. 2000; 53(12):1258-1267. [PubMed: 11146273]

16. Department of Health and Human Services. Is an activity research involving human subjects covered by 45 CFR part 46?. 2004. Available from http://www.hhs.gov/ohrp/policy/checklists/ decisioncharts.html\#c1. Accessed November 8, 2015.

17. Smedley, BD., Stith, AY., Nelson, AR. Unequal treatment: confronting racial and ethnic disparities in health care. Washington, D.C: National Academies Press; 2003.

18. Waxman AJ, Mink PJ, Devesa SS, Anderson WF, Weiss BM, Kristinsson SY, McGlynn KA, Landgren $\mathrm{O}$. Racial disparities in incidence and outcome in multiple myeloma: a population-based study. Blood. 2010; 116:5501-5506. [PubMed: 20823456]

19. Ravindran A, Bartley AC, Holton SJ, Gonsalves WI, Kapoor P, Siddiqui MA, Go RS. Prevalence, incidence and survival of smoldering multiple myeloma in the United States. Blood Cancer J. 2016; 6(10):e486. [PubMed: 27768092]

20. Hsu JW, Wingard JR, Logan BR, Chitphaldithai P, Akpek G, Anderlini P, Confer JL. Race and Ethnicity Influences Collection of Granulocyte Colony-Stimulating Factor-Mobilized Peripheral Blood Progenitor Cells from Unrelated Donors, a Center for International Blood and Marrow Transplant Research Analysis. Biol Blood Marrow Transplant. 2015; 21(1):165-171. [PubMed: 25316111] 


\section{PRECIS}

In patients with multiple myeloma, treatment disparities are not completely explained by potential access barriers. Additional factors must be explored to fully explain the disparity. 


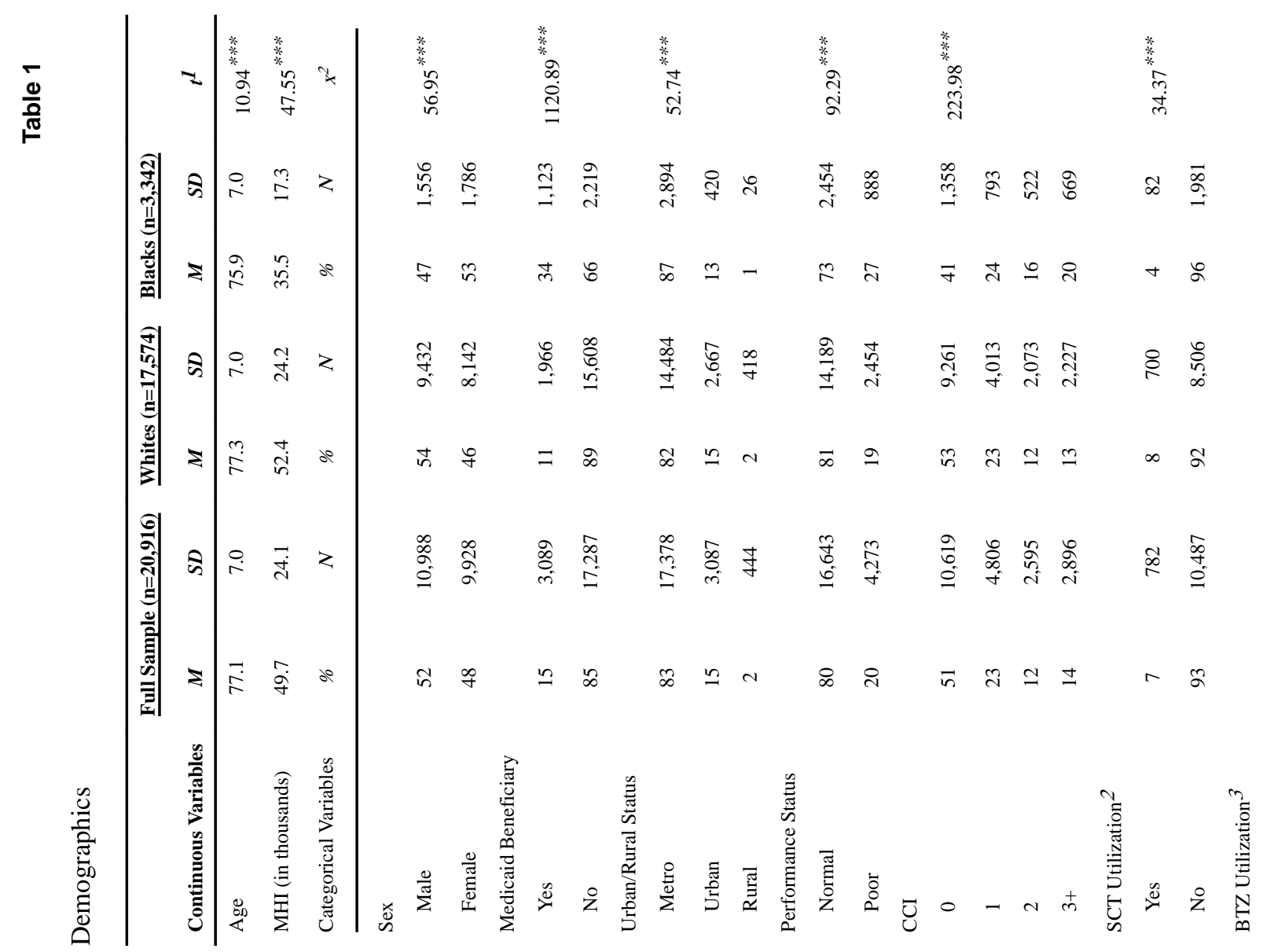




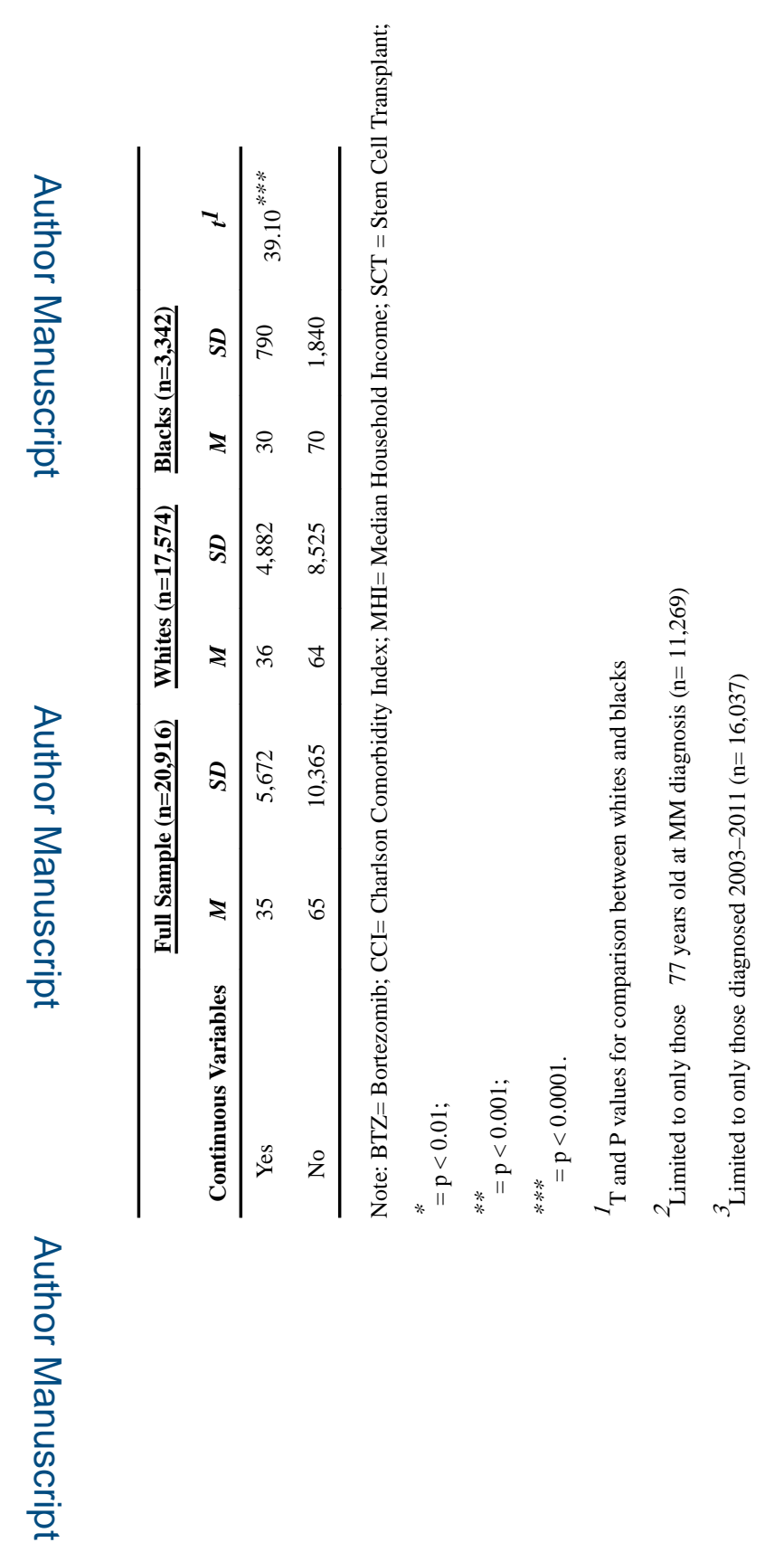






\section{를 \\ 交}

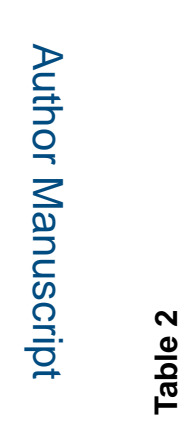

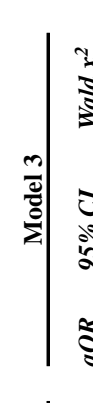



竞
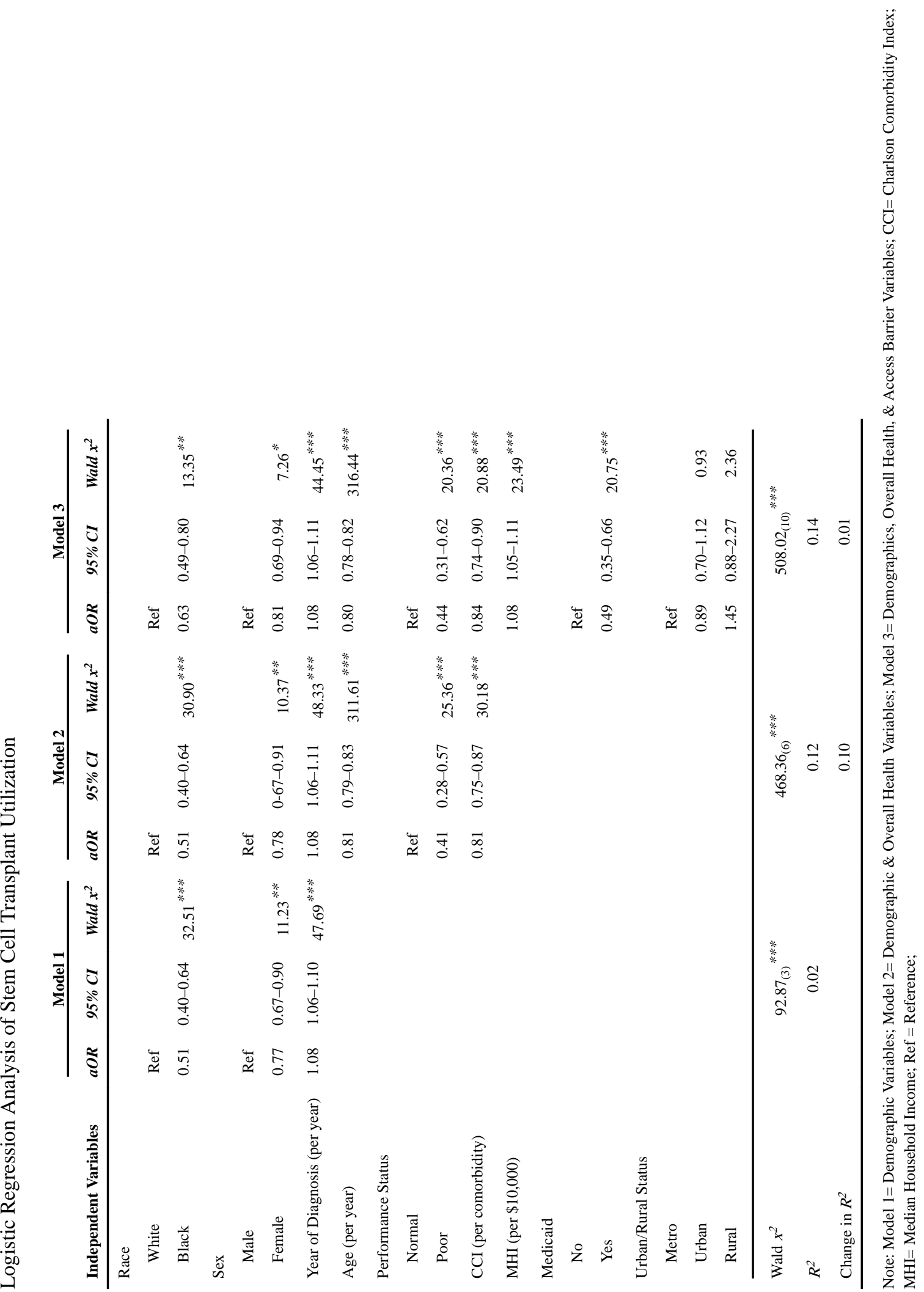

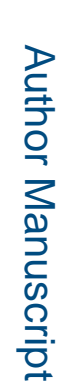

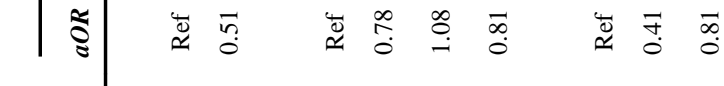

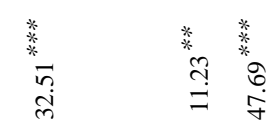



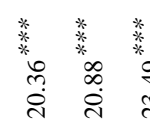

$\stackrel{*}{*}$



崩

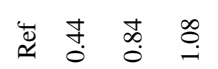

芩 $\stackrel{?}{0}$

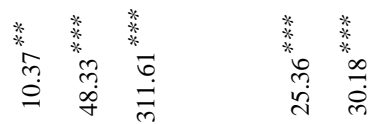

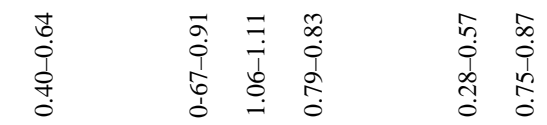

$\underset{0}{0} \div \frac{0}{0}$

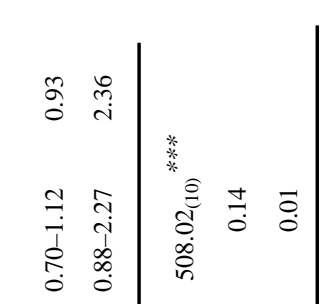

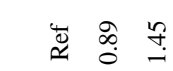

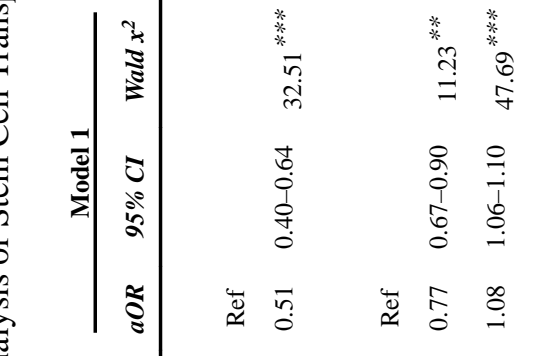

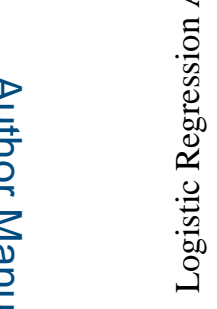

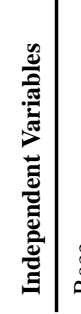

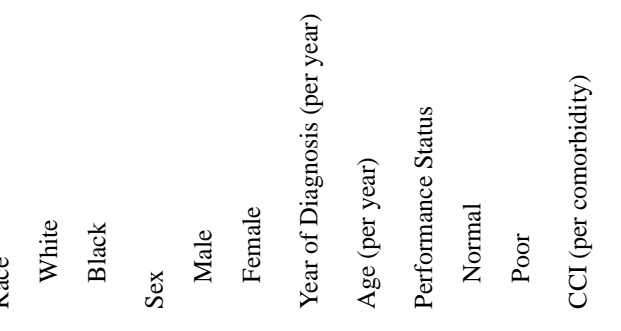

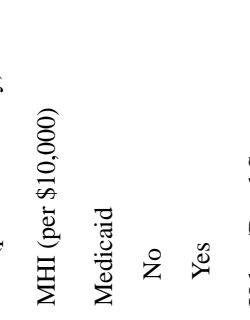

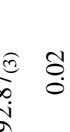



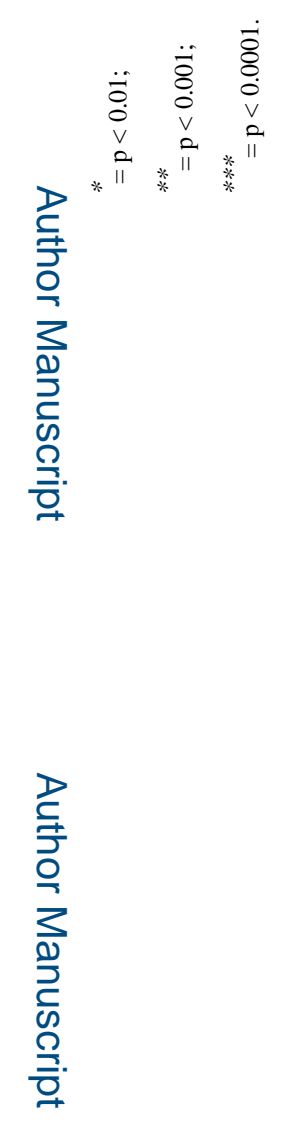

로을

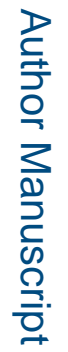

Cancer: Author manuscript; available in PMC 2018 May 01. 


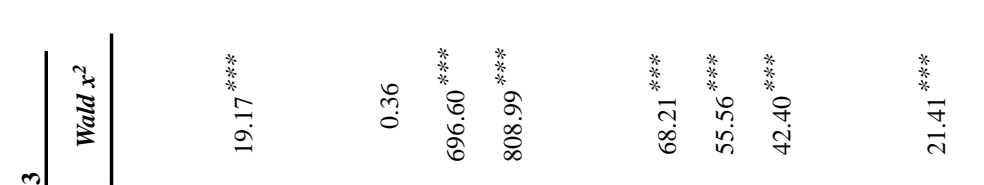
要

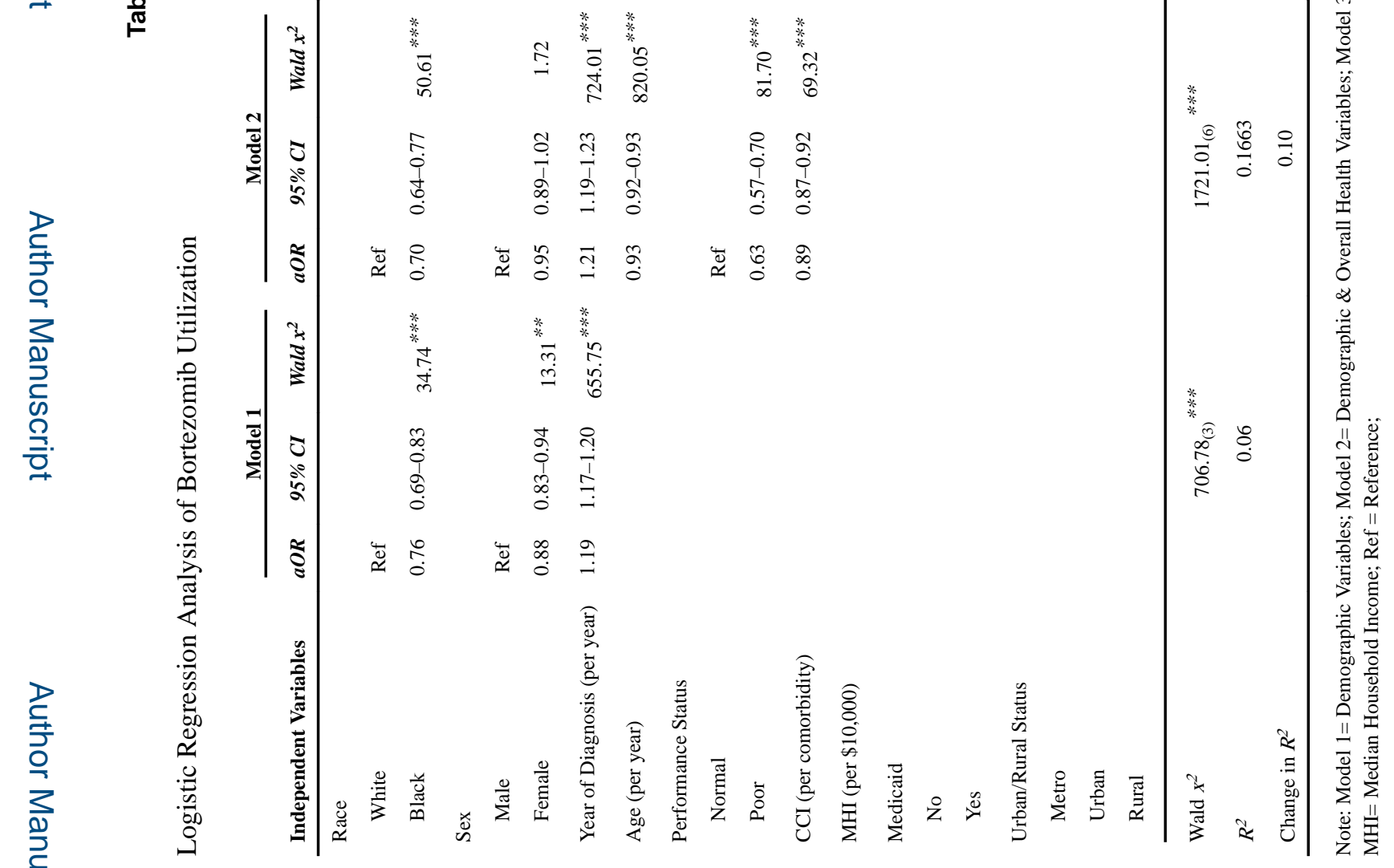



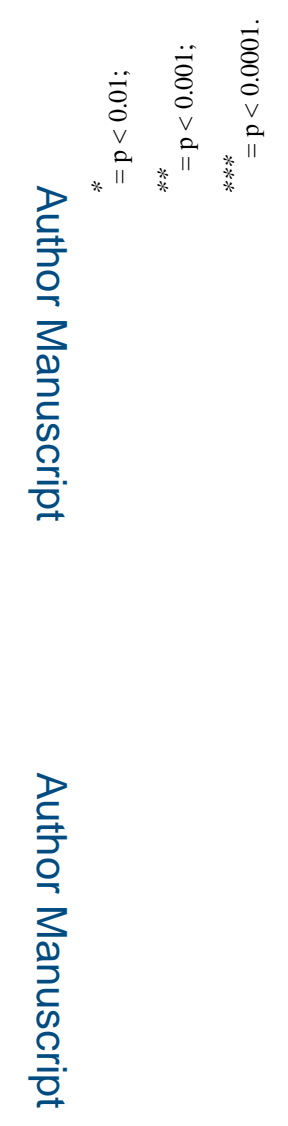

로을

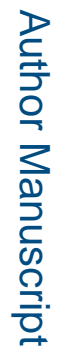

Cancer: Author manuscript; available in PMC 2018 May 01. 


\section{롤 \\ 골}

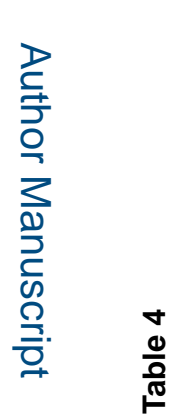

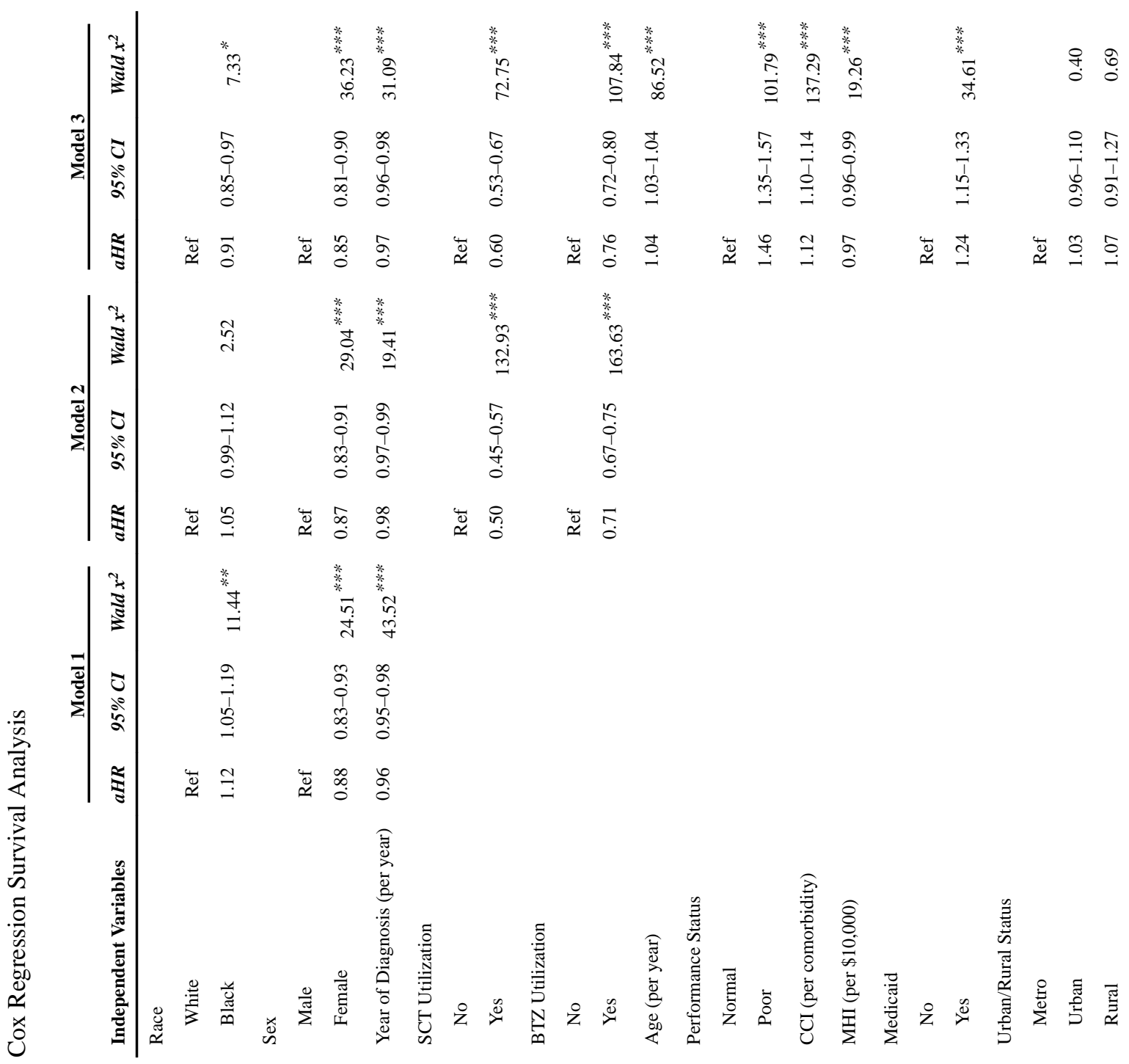

Cancer. Author manuscript; available in PMC 2018 May 01. 


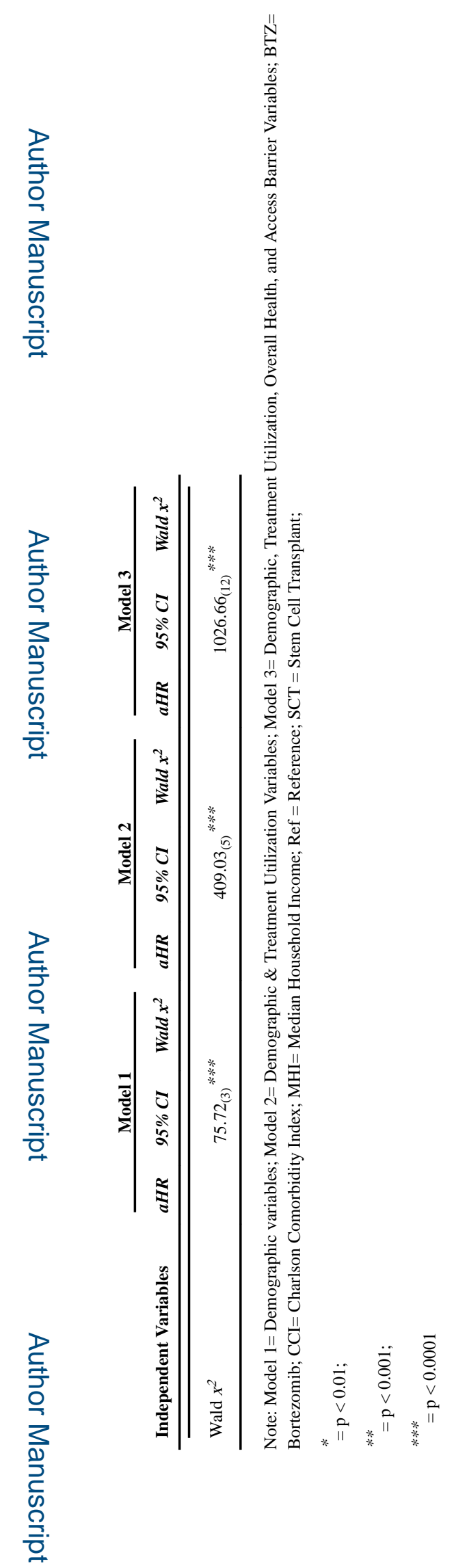

Cancer: Author manuscript; available in PMC 2018 May 01. 